\title{
New Antithrombotic Aryl-sulfonylthiosemicarbazide Derivatives Synthesized from Natural Safrole
}

\author{
Lídia M. Lima ${ }^{a, b}$, Claudia B. Ormelli ${ }^{a}$, Carlos A.M. Fraga ${ }^{a}$, \\ Ana L.P. Miranda ${ }^{a}$, and Eliezer J. Barreiro ${ }^{a} *$ \\ ${ }^{a}$ Laboratório de Avaliação e Síntese de Substâncias Bioativas, Faculdade de \\ Farmácia, Universidade Federal do Rio de Janeiro, C.P. 68006, \\ 21944-970 Rio de Janeiro - RJ, Brazil \\ ${ }^{\mathrm{b}}$ Instituto de Química, Universidade Federal do Rio de Janeiro, \\ Rio de Janeiro - RJ, Brazil
}

\begin{abstract}
No âmbito de uma linha de pesquisas que trata da síntese e avaliação farmacológica de novos candidatos a protótipos de agentes antitrombóticos, explorando a hibridação molecular como estratégia de planejamento estrutural, descrevemos neste trabalho a síntese e avaliação farmacológica de novas sulfonilsemicarbazidas $(\underline{10 \mathrm{a}-\mathrm{d}})$, planejadas por analogia estrutural a antagonistas de receptores de TXA2, utilizando o safrol (2), produto natural brasileiro abundante, isolado do óleo de Sassafrás, como matéria-prima sintética.

A avaliação das propriedades antiagregantes plaquetárias das sulfonilsemicarbazidas $(\underline{10 a-d})$ no modelo induzido por ADP, colágeno, ácido araquidônico e U46619, permitiu evidenciar um importante perfil antitrombótico ao nível da cascata do ácido araquidônico para o derivado 6-metil-3,4-metilenodioxifenil-sulfonil- $N$-feniltiosemicarbazida $(\underline{10 \mathrm{~d}})$, que representa um novo protótipo de agentes anti-trombóticos.
\end{abstract}

As part of a research program aiming at the synthesis and pharmacological evaluation of novel lead-compounds exploring Brazilian abundant natural products, we describe herein the synthesis and the antithrombotic profile of new aryl-sulfonylsemicarbazides and aryl-sulfonylthiosemicarbazides $(\underline{10 \mathrm{a}-\mathrm{d}})$. The new derivatives, designed with basis on the molecular hybridization concept, were prepared in good yields from natural safrole (9), isolated from sassafras oil.

The anti-aggregating activity of these new derivatives $(\underline{10 a-d})$ on platelet aggregation induced by ADP, collagen, arachidonic acid and U-46619, indicates an important antithrombotic profile for the 6-methyl-3,4-methylenedioxyphenyl-sulfonyl-N-phenylthiosemicarbazide derivative (10d), acting at the arachidonic acid cascade and representing a new lead-compound with antithrombotic activity.

Keywords: safrole in synthesis; phenylsulfonylthiosemicarbazide derivative; antithrombotic activity

\section{Introduction}

Thromboxane $\mathrm{A}_{2}\left(\mathrm{TXA}_{2}\right)(\underline{1})$ is one of the most potent naturally occurring platelet activators and smooth muscle constrictor substances known shows similar activity to, albeit more potent than, the precursor prostaglandin endoperoxide $\mathrm{H}_{2}\left(\mathrm{PGH}_{2}\right)(\underline{2})^{1}$, which is also the precursor of the natural anti-aggregating prostacyclin ( $\underline{3})($ Chart 1$)$. TXA 2 is a potent inductor of the platelet aggregation and constric-

*Dr. Eliezer J. Barreiro - LASSBio - UFRJ, C.P. 68006, 21944-910 Rio de Janeiro - RJ, Brazil. Phone number: +(55)(21) 2609192, ext. 220/223/238; Fax number: +(55)(21)260-2299; e-mail: eliezer@pharma.ufrj.br tion of vascular and respiratory smooth muscles ${ }^{2}$. It is considered an important chemical mediator of several physiopathological processes, e.g., thrombosis and bronchial asthma ${ }^{3,4}$. The corresponding $\mathrm{TXA}_{2}$ receptor has recently been isolated and sequenced ${ }^{5,6}$. Efforts to modulate the actions of $\mathrm{TXA}_{2}$ have focused on agents which would either inhibit the biosynthesis of $\mathrm{TXA}_{2}\left(\mathrm{TXA}_{2}\right.$ synthase inhibitor/TXS-I) or alternatively block the actions of $\mathrm{TXA}_{2}$ at the receptor level $\left(\mathrm{TXA}_{2}\right.$ receptor antagonists/TPant) ${ }^{7}$. 
Another alternative approach is the use of dual acting TPant/TXS-I ${ }^{8}$. A number of TPant have been recently described and these compounds are either structurally related to $\mathrm{TXA}_{2} / \mathrm{PGH}_{2}$, such as SQ $29.548(\underline{4})^{9,10}$, SQ 27.825 $(\underline{5})^{11}$ and S-145 $(\underline{6})^{12}$ or structurally dissimilar such as sulotroban $(\underline{7})^{13}$ (Chart 2). Structure activity relationships (SAR) concerning TPant are relatively well-known, and are based on the presence of a hydrogen bond acceptor group, hydrophobic moiety and ionic interaction site ${ }^{14,15}$.

As part of an ongoing research program devoted to identifying new antithrombotic candidates on the basis of the structure-activity relationships of TP antagonists, we described previously a new series of sulotroban ana- logues ${ }^{16}$, e.g. derivative ( $\left.\underline{8}\right)$, exploring natural safrole ( $\left.\underline{9}\right)$, an abundant Brazilian natural product occurring in the Sassafras oil ${ }^{17,18}$, as starting material. Thus, we describe herein the planning, the synthesis and the anti-platelet properties of new aryl-sulfonylsemicarbazide derivatives with the general structure $(\underline{10})$, which were structurally planned as a hybrid of both SQ 29.548 (ㄴ) and sulotrobanrelated derivative (ㅁ) (Fig. 1).

All these new compounds (10a-d) were structurally designed in order to explore a new possible bioisosteric relationship between the 1,3-benzodioxole ring, present in the natural product used as starting material, and the oxabicyclo[2.2.1] heptane system of (ㄴ-6), considering the hydro-<smiles>CCCCCC/C=C/C[C@H]1C2CC(O2)O[C@H]1/C=C/[C@H](O)CCCC(=O)O</smiles>

Thromboxane $A_{2}\left(T_{X A}, 1\right)$
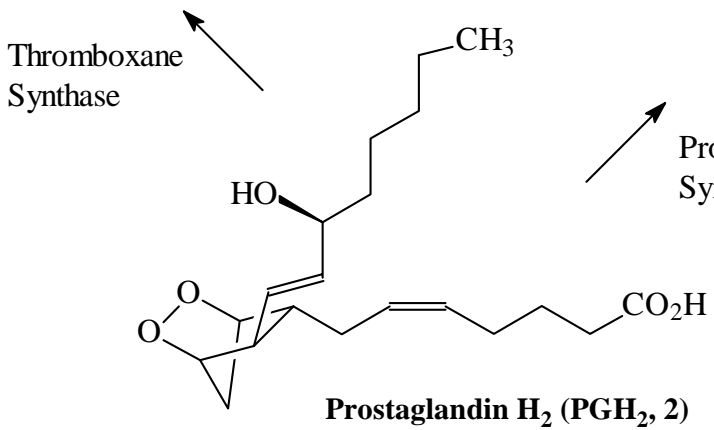

Chart 1.
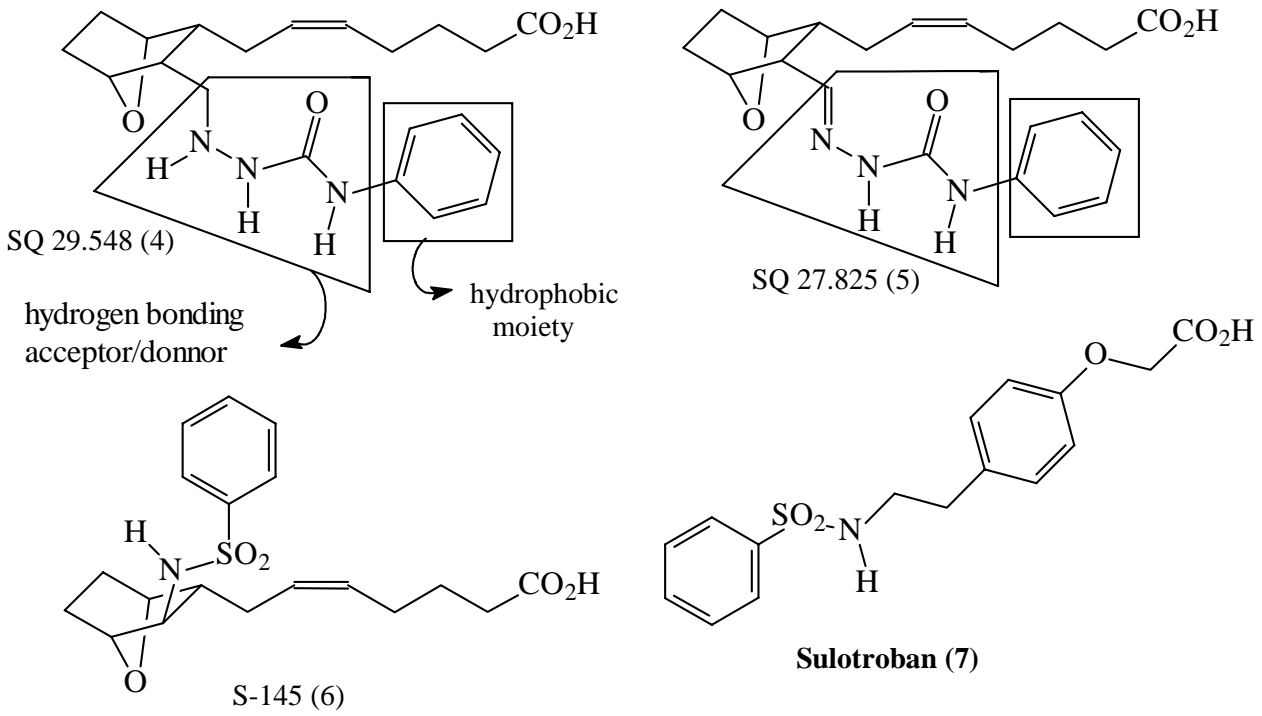

Sulotroban (7)

Chart 2. 
gen-bonding acceptor character of both subunits as represented by the oxygen atom indicated in Figure 1. The introduction of the sulfonyl group on the semicarbazide side chain, was performed with the aim to study the eventual contribution of this multiple hydrogen-bonding acceptor/donor functionality in the antiplatelet activity of these new compounds (10a-d).

\section{Results and Discussion}

The new target derivatives, aryl-sulfonylsemicarbazides, $(10 \mathrm{a}-\mathrm{d})$ were synthesized as depicted in Scheme 1. Our synthetic approach to these new compounds identified 6methyl-3,4-methylenedioxy-phenylsulfonyl chloride (14) as the key intermediate (Scheme 1). This compound can be obtained by regioselective aromatic electrophilic substitution at the C-6 position of the precursor 3,4-methylenedioxytoluene $(\underline{12})$, which was obtained in $c a .50 \%$ overall yield from natural safrole (2). Simple distillation of Sassafras oil yielded 9 in $85 \%$ yield. Base catalysed isomerization of the double bond followed by oxidative cleavage and Wolff-Kishner reduction yielded $12^{19-22}$. The arylsulfonyl chloride derivative (14) was next prepared in two steps from (12), applying methodology previously used in this laboratory to promote an efficient and mild sulfonation of the sensitive 1,3-benzodioxole derivatives ${ }^{16,20}$. Thus, treatment of (12) with a mixture of acetic anhydride in ethyl acetate, containing $1.1 \mathrm{eq}$. of sulfuric acid, at $0{ }^{\circ} \mathrm{C}$, followed by careful addition of a solution of potassium acetate in ethanol, furnished as the only product, the potassium salt of 6-methyl-3,4-methylenedioxy-phenylsulfonic acid (13) in $91 \%$ yield. The analysis of ${ }^{1} \mathrm{H}-\mathrm{NMR}$ spectra of compound (13), confirmed the anticipated regioselectivity of this process as evidenced by the presence of two singlet signals at $\delta 6.70$ and 7.27. The next step in the planned synthetic route was the treatment of the potassium salt (13) with thionyl chloride, catalyzed by the Vilsmeyer-Haack complex, to afford the desired arylsulfonyl chloride derivative (14) in 74\% yield $^{16,19,20}$.

With an attractive method for access to the key intermediate (14) in hand, we next performed the condensation step of this compound with $40 \%$ aqueous hydrazine hydrate in chloroform at $0{ }^{\circ} \mathrm{C}$ to obtain the corresponding arylsulfonylhydrazine (15) in $92 \%$ yield $^{16,18}$. The infrared spectrum of (15) indicated the sulfonylhydrazine moiety by the presence of absorptions at 3398, 3370 and $3327 \mathrm{~cm}^{-1}$, typical for the group -NH-NH . Finally, the synthesis of the novel arylsulfonylsemicarbazide (10a-d) was concluded in good yield (Table 1), by treatment of the arylsulfonylhydrazine derivative $(\underline{15})$ with phenylisocyanate, 4-chlorophenylisocyanate, 4-bromophenylisocyanate and phenylisothiocyanate, respectively, in tetrahydrofuran at room temperature ${ }^{23}$ (Scheme 1). The ${ }^{13} \mathrm{C}$-NMR spectrum of compounds (10a-d) (Table 2) shows the $\mathrm{C}=\mathrm{O}$ and $\mathrm{C}=\mathrm{S}$ signals at $\delta 154.7$ and 180.8 , corresponding to semicarbazide and thiosemicarbazide moieties, respectively.

The antithrombotic activity of these novel arylsulfonylsemicarbazide derivatives (10a-d) was evaluated by their ability to inhibit platelet aggregation of rabbit platelet-rich
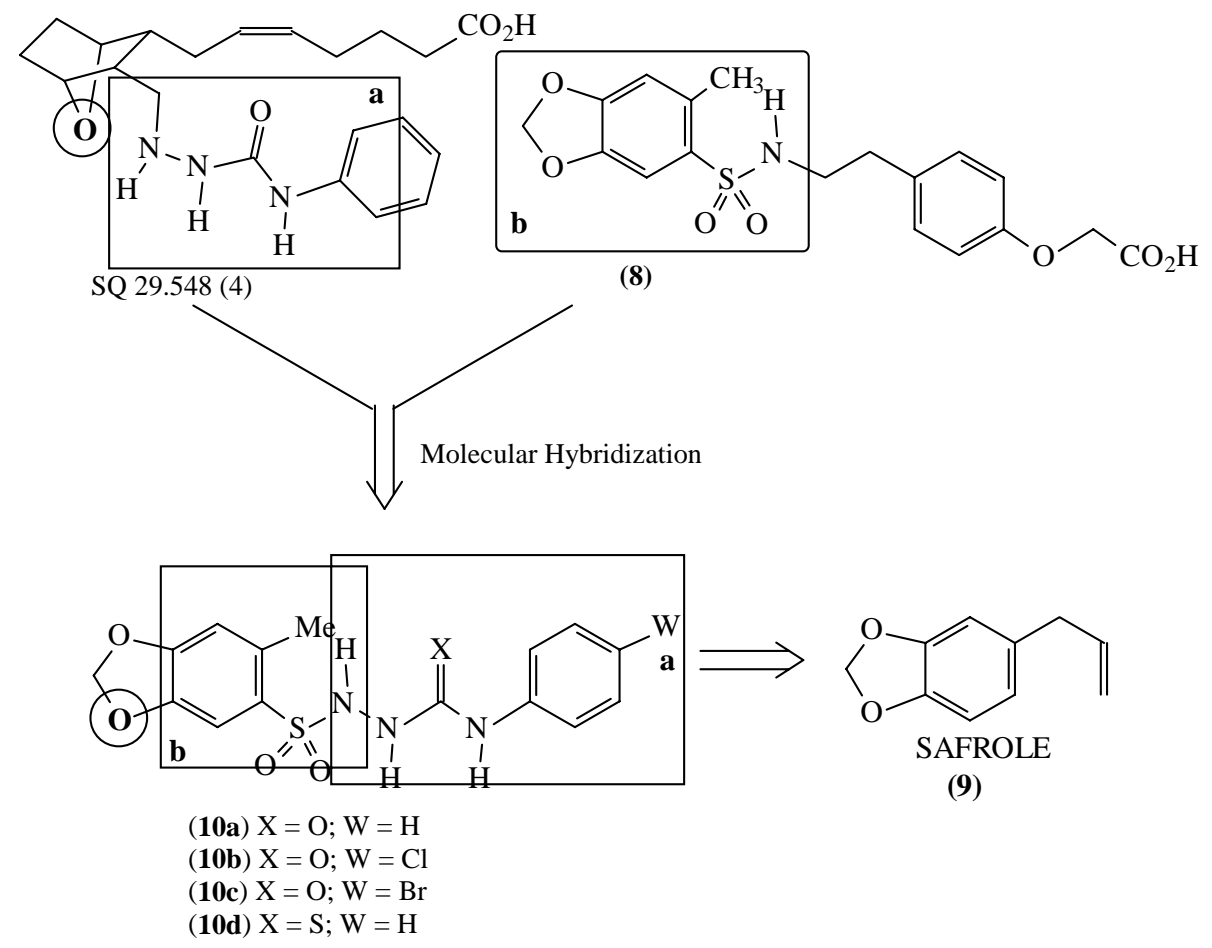

Figure 1. Design concept of new TPant derivatives (10a-d). 
Table 1. 6-Methyl-3,4-methylenedioxyphenylsulfonyl- $N$-phenylsemicarbazide (10a-d).<smiles>[Y1]c1ccc(NC([X])NNS(=O)(=O)c2cc3c(cc2C)OCO3)cc1</smiles>

(10a-d)

\begin{tabular}{lllcccc}
\hline \multirow{2}{*}{ Compound } & \multicolumn{2}{c}{ Substituents } & Molecular formula & Yield (\%) & Molecular weight & m.p $\left({ }^{\circ} \mathrm{C}\right)$ \\
\cline { 2 - 3 } & $\mathrm{X}$ & $\mathrm{W}$ & & & & \\
\cline { 2 - 5 }$\underline{10 \mathrm{a}}$ & $\mathrm{O}$ & $\mathrm{H}$ & $\mathrm{C}_{15} \mathrm{H}_{15} \mathrm{~N}_{3} \mathrm{O}_{5} \mathrm{~S}$ & 85 & 349.36 & $185-186$ \\
$\underline{10 \mathrm{~b}}$ & $\mathrm{O}$ & $\mathrm{Cl}$ & $\mathrm{C}_{15} \mathrm{H}_{14} \mathrm{ClN}_{3} \mathrm{O}_{5} \mathrm{~S}$ & 75 & 383.80 & $201-202$ \\
$\underline{10 \mathrm{c}}$ & $\mathrm{O}$ & $\mathrm{Br}$ & $\mathrm{C}_{15} \mathrm{H}_{14} \mathrm{BrN}_{3} \mathrm{O}_{5} \mathrm{~S}$ & 92 & 428.25 & $214-216$ \\
$\underline{10 \mathrm{~d}}$ & $\mathrm{~S}$ & $\mathrm{H}$ & $\mathrm{C}_{15} \mathrm{H}_{15} \mathrm{~N}_{3} \mathrm{O}_{4} \mathrm{~S}_{2}$ & 80 & 365.42 & $163-165$ \\
\hline
\end{tabular}

plasma (PRP) induced by adenosine diphosphate (ADP, 5 $\mu \mathrm{M})$, or collagen $(90 \mu \mathrm{M})$, or arachidonic acid (AA, 200 $\mu \mathrm{M})$ or U-46619 (3 $\mu \mathrm{M})$, a stable analogue of TXA 2 , used as a pharmacological probe to TP. The results of the antiplatelet profile of $(\underline{10 a-d})$ are displayed in Table 3 .

Consistent with these compounds exerting their effects at the arachidonic acid cascade level was that none of them were effective in preventing platelet aggregation induced by ADP. Compound (10d), belonging to the phenylthiosemicarbazide class, was able to significantly inhibit the platelet aggregation induced by collagen (25.9\%) at $90 \mu \mathrm{M}$ concentration. In contrast, this compound was not able to promote any platelet inhibition when induced by arachidonic acid. It is known that in rabbit PRP, the collagen induced aggregation is partially mediated by $\mathrm{TXA}_{2}$ formation ${ }^{24}$. Thus, in order to evaluate the activity profile of these compounds at different concentrations, we decided to investigate the biological response at $200 \mu \mathrm{M}$ concentration. At this concentration, compound $(\underline{10 \mathrm{~d}})$ presented a very important anti-platelet profile, inhibiting by $99.8 \%$ and $49.1 \%$ the aggregation induced by arachidonic acid and collagen respectively. These results indicate an antithrombotic activity at the arachidonic acid cascade level, and consequently it was decided to evaluate the effect of these compounds on the platelet aggregation induced by $\mathrm{U}-46619(3 \mu \mathrm{M})$, in order to verify any eventual TPant properties. In this bioassay (Table 3 ) compound (10d) effectively inhibited the platelet aggregation by $24.4 \%$ at a<smiles>C=CC(C#CCc1ccc2c(c1)OCO2)[C@H]1Oc2ccc(C(=O)NC3COc4ccc(C)cc4O3)cc2O1</smiles>

(9)

(11)<smiles>Cc1cc2c(cc1S(=O)(=O)NN)OCO2</smiles>

(15)

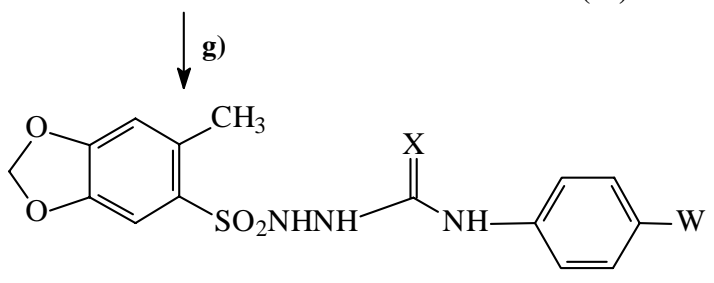

(12)<smiles>Cc1cc2c(cc1S(=O)(=O)O)OCO2</smiles>

(13)

Scheme 1. a) $3 \mathrm{~N} \mathrm{KOH} / n \mathrm{BuOH}$, reflux, $6 \mathrm{~h}, 96 \%$; b) 1$) \mathrm{O}_{2} / \mathrm{O}_{3}, \mathrm{AcOH}, 0{ }^{\circ} \mathrm{C}$; 2) $\mathrm{Zn}, \mathrm{AcOH}, 0{ }^{\circ} \mathrm{C}, 4 \mathrm{~h}, 70 \%$; c) $\mathrm{KOH}, \mathrm{N}_{2} \mathrm{H}_{4} \cdot \mathrm{H}_{2} \mathrm{O}$, ethyleneglicol, reflux, $6 \mathrm{~h}, 73 \%$; d) 1) $\mathrm{H}_{2} \mathrm{SO}_{4}, \mathrm{Ac}_{2} \mathrm{O}$, AcOEt, $0{ }^{\circ} \mathrm{C}$; 2) $\mathrm{AcOK}$, EtOH. r.t., 30 min., $91 \%$; e) $\mathrm{SOCl}_{2}, \mathrm{DMF}_{\text {(cat.) }}$ reflux, 4 h, $74 \%$; f) $40 \%$ aq. $\mathrm{N}_{2} \mathrm{H}_{4} . \mathrm{H}_{2} \mathrm{O}, \mathrm{CHCl}_{3}$, $\left.0{ }^{\circ} \mathrm{C}, 2 \mathrm{~h}, 92 \% ; \mathbf{g}\right) 4-\mathrm{W}-\mathrm{C}_{6} \mathrm{H}_{4} \mathrm{NCX}, \mathrm{THF}$, r.t., $12 \mathrm{~h}$. 
Table 2. ${ }^{13} \mathrm{C}$ nuclear magnetic shifts of compounds $(\underline{10 \mathrm{a}-\mathrm{d}})^{\mathrm{a}}$ at $50 \mathrm{MHz}$.

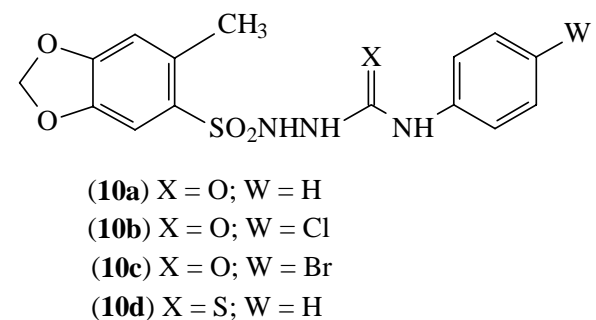

\begin{tabular}{|c|c|c|c|c|}
\hline & \multicolumn{4}{|c|}{ Compounds } \\
\hline & $\underline{10 \mathrm{a}}$ & $10 \mathrm{~b}$ & $\underline{10 \mathrm{c}}$ & $\underline{10 \mathrm{~d}}$ \\
\hline $\mathrm{C}-1$ & 134.43 & 134.32 & 134.30 & 134.79 \\
\hline $\mathrm{C}-2$ & 99.82 & 99.71 & 99.70 & 99.83 \\
\hline $\mathrm{C}-3$ & 145.15 & 145.13 & 145.09 & 145.32 \\
\hline $\mathrm{C}-4$ & 150.91 & 150.91 & 150.88 & 151.21 \\
\hline $\mathrm{C}-5$ & 112.00 & 111.92 & 111.90 & 111.99 \\
\hline C-6 & 128.81 & 128.70 & 128.78 & 128.22 \\
\hline $\mathrm{C}-1^{\prime}$ & 139.20 & 138.31 & 138.65 & 138.78 \\
\hline $\mathrm{C}-2^{\prime}$ & 118.60 & 120.9 & 120.53 & 125.07 \\
\hline $\mathrm{C}-3^{\prime}$ & 128.63 & 128.40 & 131.28 & 128.00 \\
\hline C-4' & 122.11 & 125.61 & 113.53 & 124.82 \\
\hline $\mathrm{O}_{\underline{C}} \mathrm{H}_{2} \mathrm{O}$ & 92.21 & 92.20 & 92.17 & 92.29 \\
\hline $\mathrm{Ar}^{2} \mathrm{H}_{3}$ & 20.14 & 20.08 & 20.08 & 20.29 \\
\hline HNCONH & 154.70 & 154.71 & 154.64 & \\
\hline HNCSNH & --- & --- & --- & 180.86 \\
\hline
\end{tabular}

${ }^{\mathrm{a}}$ ca. $60 \mathrm{mg}$ of compound in $0.7 \mathrm{~mL}$ of DMSO-d6.

$200 \mu \mathrm{M}$ concentration in this assay. These results seem to indicate that $(\underline{10 \mathrm{~d}})$ does not act at the TP level but does prevent platelet aggregation by acting upon the arachidonic acid cascade, probably at the TXS level. It is important to mention that compound $(\underline{10 \mathrm{c}})$, belonging to the paraphenylsemicarbazide substituted class, at $200 \mu \mathrm{M}$ concentration, was the most active one $(26 \%)$ in the assay induced by U-46619. The activity presented by both para-halogenated phenylsemicarbazide derivatives $(\underline{10 \mathrm{~b}}$ and $\underline{10 \mathrm{c}})$, in comparison to the effect presented by the derivative (10a), seems to indicate that the presence of an halogen atom at the para-position of the phenyl ring of the semicarbazide moiety could improve the anti-platelet activity, in the assay induced by U-46619, in this series of derivatives.

\section{Conclusions}

As concluding remarks, the synthetic route described herein for access to these new aryl-sulfonylsemicarbazide and phenylsulfonylthiosemicarbazide derivatives (10a-d), structurally planned as hybrids of known TPant compounds, possessing the semicarbazide group (e.g. SQ29548, 4), represent a useful, efficient and high yield
Table 3. 
Table 3. Effect of sulfonyl semicarbazides derivatives $(\underline{10 \mathrm{a}-\mathrm{d}})$ on in vitro platelet aggregation of citrated rabbit platelet-rich plasma induced by arachidonic acid, ADP, collagen and U46619.

\begin{tabular}{|c|c|c|c|c|c|c|c|c|c|c|c|c|c|}
\hline \multirow[t]{2}{*}{ Compounds } & \multirow[t]{2}{*}{$\begin{array}{c}\text { Concentration } \\
(\mu \mathrm{M}) \\
\end{array}$} & \multirow[b]{2}{*}{$\mathrm{N}$} & \multicolumn{2}{|c|}{$\begin{array}{c}\text { Arachidonic Acid } \\
(200 \mu \mathrm{M})\end{array}$} & \multirow[b]{2}{*}{$\mathrm{n}$} & \multicolumn{2}{|c|}{$\begin{array}{c}\text { ADP } \\
(5 \mu \mathrm{M})\end{array}$} & \multirow[b]{2}{*}{$\mathrm{n}$} & \multicolumn{2}{|c|}{$\begin{array}{l}\text { Collagen } \\
(5 \mu \mathrm{g} / \mathrm{mL})\end{array}$} & \multirow[b]{2}{*}{$\mathrm{n}$} & \multicolumn{2}{|c|}{$\begin{array}{c}\mathrm{U}-46619 \\
(3 \mu \mathrm{M})\end{array}$} \\
\hline & & & $\begin{array}{c}\text { Aggregation } \\
(\%)\end{array}$ & $\begin{array}{c}\text { Inhibition } \\
(\%)\end{array}$ & & $\begin{array}{c}\text { Aggregation } \\
(\%)\end{array}$ & $\begin{array}{c}\text { Inhibition } \\
(\%)\end{array}$ & & $\begin{array}{c}\text { Aggregation } \\
\text { (slope) }\end{array}$ & $\begin{array}{c}\text { Inhibition } \\
(\%)\end{array}$ & & $\begin{array}{c}\text { Aggregation } \\
(\%)\end{array}$ & $\begin{array}{c}\text { Inhibition } \\
(\%)\end{array}$ \\
\hline Control & - & 4 & $73.1 \pm 3.3$ & - & 5 & $45.4 \pm 3.9$ & - & 6 & $11.2 \pm 0.3$ & - & 7 & $61.4 \pm 3.3$ & - \\
\hline Indomethacin & 9 & 4 & - & $90.0 *$ & 3 & - & 1.2 & 4 & - & $94.8 *$ & 5 & - & 1.0 \\
\hline \multirow[t]{2}{*}{$\underline{10 \mathrm{a}}$} & 90 & 3 & $65.9 \pm 6.4$ & 9.8 & 3 & $39.7 \pm 0.3$ & 12.5 & 3 & $9.1 \pm 0.3$ & 9.8 & 3 & $53.5 \pm 1.0$ & 12.9 \\
\hline & 200 & & & & & & & & & & 3 & $49.9 \pm 5.0$ & \\
\hline \multirow[t]{2}{*}{$\underline{10 b}$} & 90 & 3 & $63.4 \pm 4.3$ & 13.3 & 3 & $42.1 \pm 2.7$ & 7.3 & 3 & $8.9 \pm 0.9$ & 20.5 & 3 & $48.9 \pm 1.4$ & $20.3 *$ \\
\hline & 200 & & & & & & & & & & 3 & $46.8 \pm 4.4$ & \\
\hline \multirow[t]{2}{*}{$\underline{10 c}$} & 90 & 3 & $64.3 \pm 4.7$ & 12.0 & 3 & $42.3 \pm 0.9$ & 6.8 & 3 & $9.2 \pm 0.7$ & 8.9 & 3 & $52.1 \pm 2.7$ & 15.1 \\
\hline & 200 & & $67.8 \pm 7.1$ & 9.0 & & & & & & & 3 & $45.4 \pm 6.3$ & $26.0 *$ \\
\hline \multirow[t]{2}{*}{$\underline{10 d}$} & 90 & 3 & $61.7 \pm 6.0$ & 15.6 & 3 & $45.2 \pm 1.4$ & 0.4 & 3 & $8.3 \pm 0.8$ & $25.9 *$ & 5 & $52.9 \pm 8.5$ & 13.8 \\
\hline & 200 & 3 & $0.13 \pm 0.03$ & $99.8 *$ & & & & 3 & $5.7 \pm 0.6$ & $49.1 *$ & 3 & $46.4 \pm 2.8$ & $24.4 *$ \\
\hline
\end{tabular}

$\mathrm{n}$ - number of independent experiments carried out in triplicate. * $\mathrm{p}<0.05$ compared to appropriate control (Student's "t" test). 
method for exploring safrole (2), an abundant Brazilian natural product, as starting material. The pharmacological results identify a potent new anti-platelet compound, represented by the phenylsulfonylthiosemicarbazide derivative $(\underline{10 \mathrm{~d}})$, that can be considered as a new lead-candidate to antithrombotic agents acting at the arachidonic acid cascade level.

\section{Experimental}

\section{Chemistry}

Melting points were determined with a Thomas-Hoover apparatus and are uncorrected. Proton magnetic resonance $\left({ }^{1} \mathrm{H}-\mathrm{NMR}\right)$ spectra were determined in deuterated solvents, using tetramethylsilane as an internal standard with a Brucker AC 200 spectrometer. Splitting patterns were as follows: s, singlet; d, doublet; $\mathrm{m}$, multiplet. The carbon magnetic resonance spectra $\left({ }^{13} \mathrm{C}-\mathrm{NMR}\right)$ were determined in the same spectrometer described above at $50 \mathrm{MHz}$, using deuterated chloroform as internal standard. Infrared spectra (IR) were obtained with a Perkin-Elmer 1600 spectrometer as neat film and $\mathrm{KBr}$ pellets. The mass spectra (MS) were obtained on a GC/VG Micromass 12 at $70 \mathrm{eV}$.

The progress of all reactions was monitored by TLC which was performed on $2.0 \mathrm{~cm}$ x $5.0 \mathrm{~cm}$ aluminum sheets precoated with silica gel 60 (HF-254, Merck) to a thickness of $0.25 \mathrm{~mm}$. The developed chromatograms were visualized under ultraviolet light (254-265 nm). For column chromatography Merck silica gel (70-230 mesh) was used. Solvents used in reactions were dried, redistillated prior to use and stored over 3-4 $\AA$ molecular sieves.

\section{Potassium 6-methyl-3,4-methylenedioxybenzenesulfonate} $(\underline{13})^{16,19-20}$

To a solution of 3,4-methylenedioxytoluene (12) $(0.1 \mathrm{~g}$; $0.74 \mathrm{mmol})$ in $2.2 \mathrm{~mL}$ of ethyl acetate containing acetic anhydride $(0.21 \mathrm{~mL} ; 2.2 \mathrm{mmol})$ cooled at $0{ }^{\circ} \mathrm{C}$, was added, dropwise, a solution of sulfuric acid $(0.06 \mathrm{~mL} ; 0.93 \mathrm{mmol}$; $\mathrm{d}=1.84$ ) in ethyl acetate. To this reaction mixture, after stirring for $2 \mathrm{~h}$ at room temperature, was next added a solution of potassium acetate $(0.11 \mathrm{~g} ; 1.1 \mathrm{mmol})$ in ethanol $(0.6 \mathrm{~mL})$ and the suspension formed was maintained under stirring at room temperature for an additional $30 \mathrm{~min}$. The resulting precipitates were removed by filtration, washed with ethyl acetate to give $0.16 \mathrm{~g}(91 \%)$ of $(\underline{13})$ as colorless powder, mp $189^{\circ} \mathrm{C} .{ }^{1} \mathrm{H}-\mathrm{NMR}\left(\mathrm{D}_{2} \mathrm{O}\right) \delta: 2.45\left(\mathrm{~s}, 3 \mathrm{H}, \mathrm{ArCH}_{3}\right)$, 5.89 (s, 2H, OC$\left.{ }_{2} \mathrm{O}\right), 6.70$ (s, $\left.1 \mathrm{H}, \mathrm{Ar}-\mathrm{H}_{5}\right), 7.27(\mathrm{~s}, 1 \mathrm{H}$, Ar-H $)_{2}$ ppm; IR (KBr): 1346 ( $v$ S-O), 1242 ( $v$ C-O), 955 (v S-O-C) $\mathrm{cm}^{-1}$.

\section{6-Methyl-3,4-methylenedioxyphenylsulfonyl chloride $(\underline{14})^{16,19-20}$}

A mixture of thionyl chloride $(3.9 \mathrm{~mL} ; 53.4 \mathrm{mmol})$ and dry dimethylformamide $(0.1 \mathrm{~mL})$ was added to the dry potassium salt derivative (13) $(2.5 \mathrm{~g} ; 9.2 \mathrm{mmol})$. The reaction mixture, maintained under a nitrogen atmosphere, was stirred at reflux for $4 \mathrm{~h}$ and then poured into crushed ice and extracted with methylene chloride $(5 \times 30 \mathrm{~mL})$. The organic extracts were dried over anhydrous $\mathrm{Na}_{2} \mathrm{SO}_{4}$ and evaporated at reduced pressure to give $1.8 \mathrm{~g}$ (74\%) of the sulfonyl chloride derivative $(\underline{14})$ as yellow prisms: mp $79{ }^{\circ} \mathrm{C} .{ }^{1} \mathrm{H}-$ $\operatorname{NMR}\left(\mathrm{CDCl}_{3}\right) \delta: 2.70\left(\mathrm{~s}, 3 \mathrm{H}, \mathrm{ArCH}_{3}\right), 6.9\left(\mathrm{~s}, 2 \mathrm{H}, \mathrm{OCH}_{2} \mathrm{O}\right)$, 6.81 (s, 1H, Ar-H $), 7.49$ (s, 1H, Ar-H $)$ ppm; IR (KBr): 1359 (v S-O), 1251 ( $v$ C-O), 1160 (v S-O-C) $\mathrm{cm}^{-1}$.

\section{6-Methyl-3,4-methylenedioxyphenylsulfonylhydrazide} $(\underline{15})^{16}$

To a solution of sulfonyl chloride derivative (14) $(0.4 \mathrm{~g}$; $1.7 \mathrm{mmol})$ in chloroform $(20 \mathrm{~mL})$, cooled at $0{ }^{\circ} \mathrm{C}$, was added dropwise $40 \%$ aqueous hydrazine hydrate $(5 \mathrm{~mL})$. The reaction mixture was stirred for $2 \mathrm{~h}$ at at $0{ }^{\circ} \mathrm{C}$, then poured into water $(9 \mathrm{~mL})$ and extracted with chloroform (4 x $9 \mathrm{~mL}$ ). The organic layer was washed with brine, dried over anhydrous $\mathrm{Na}_{2} \mathrm{SO}_{4}$ and evaporated at reduced pressure to give $0.36 \mathrm{~g}(92 \%)$ of the sulfonylhydrazide derivative (15) as colorless prisms: $\mathrm{mp} 120-121{ }^{\circ} \mathrm{C} .{ }^{1} \mathrm{H}-\mathrm{NMR}$ (DMSOd $\mathrm{d}_{6} \delta: 2.50\left(\mathrm{~s}, 3 \mathrm{H}, \mathrm{ArCH}_{3}\right), 6.9\left(\mathrm{~s}, 2 \mathrm{H}, \mathrm{OCH}_{2} \mathrm{O}\right), 6.97(\mathrm{~s}$, $1 \mathrm{H}, \mathrm{Ar}-\mathrm{H}_{5}$ ), 7.28 (s, 1H, Ar-H $)^{\text {ppm; }}{ }^{13} \mathrm{C}-\mathrm{NMR}$ (DMSOd 6$)$ d: $19.8\left(\mathrm{Ar}^{-} H_{3}\right), 92.1\left(\mathrm{OCH}_{2} \mathrm{O}\right), 99.9\left(\mathrm{C}_{2}\right), 111,8\left(\mathrm{C}_{5}\right)$, $128.7\left(\mathrm{C}_{6}\right), 133.2\left(\mathrm{C}_{1}\right), 145.1\left(\mathrm{C}_{3}\right), 150.4\left(\mathrm{C}_{4}\right) \mathrm{ppm}$; IR (KBr): 3398 and $3370(v \mathrm{H}-\mathrm{N}-\mathrm{H}), 3327(\mathrm{v} \mathrm{N}-\mathrm{H}), 1348$ and 1117 (v S-N), 1213 (v C-O) cm ${ }^{-1}$; MS (m/z): $230\left(\mathrm{M}^{+}\right.$, 28\%), 200 (90\%), 135 (79\%), 151 (67\%), 77 (66\%).

General procedure for obtaining 6-Methyl-3,4-methylenedioxyphenylsulfonyl-N-phenylsemicarbazide $(\underline{10 a-d})^{23}$

To a solution of $0.2 \mathrm{~g}(0.87 \mathrm{mmol})$ of sulfonylhydrazine (15) in freshly destilled tetrahydrofuran was added isocyanate derivative $(0.87 \mathrm{mmol})$. The reaction mixture was stirred for $12 \mathrm{~h}$ at room temperature, when the end of reaction was observed by tlc. Next, the arylsulfonylsemicarbazides $(\underline{10 \mathrm{a}-\mathrm{d}})$ were isolated by concentration of the reaction mixture under reduced pressure and addition of cold water to furnish the desired product as colorless prisms in $80-92 \%$ ( see Table 1).

\section{6-Methyl-3,4-methylenedioxyphenylsulfonyl-N-phenyl- semicarbazide $(\underline{10 a})$}

This compound was obtained, in $85 \%$ yield, using 0.1 mL of phenylisocyanate, as colorless prisms: mp 185-186 ${ }^{\circ} \mathrm{C}$. ${ }^{1} \mathrm{H}-\mathrm{NMR}$ (DMSO-d ${ }_{6}$ ) $\delta: 2.50$ (s, 3H, $\mathrm{ArC}_{3}$ ), 6.9 (s, $\left.2 \mathrm{H}, \mathrm{OC}_{2} \mathrm{O}\right), 6.97$ (s, 1H, Ar-H $), 7.28$ (s, 1H, Ar- $\left.\mathrm{H}_{2}\right),(\mathrm{m}$, $5 \mathrm{H}, \mathrm{Ar}-\mathrm{H}_{2},-6$ ) $\mathrm{ppm} ;{ }^{13} \mathrm{C}-\mathrm{NMR}\left(\mathrm{DMSO}_{-} \mathrm{d}_{6}\right) \quad \delta: 20.14$ $\left(\mathrm{Ar}^{\mathrm{C}} \mathrm{H}_{3}\right), 92.00\left(\mathrm{O}_{\mathrm{CH}} \mathrm{O}\right), 99.82\left(\mathrm{C}_{2}\right), 112.00\left(\mathrm{C}_{5}\right), 118.60$ $\left(\mathrm{C}_{2}\right.$, and $\left.\mathrm{C}_{6}{ }^{\prime}\right), 122.11\left(\mathrm{C}_{4}{ }^{\prime}\right), 128.63\left(\mathrm{C}_{3}\right.$, and $\left.\mathrm{C}_{5},\right), 128.81$ $\left(\mathrm{C}_{6}\right), 134.43\left(\mathrm{C}_{1}\right), 139.20\left(\mathrm{C}_{1}\right), 145.15\left(\mathrm{C}_{3}\right), 150.91\left(\mathrm{C}_{4}\right)$, $154.70(\mathrm{HNC}=\mathrm{O})$ ppm; IR (KBr): $3329\left(\mathrm{v} \mathrm{SO}_{2} \mathrm{~N}-\mathrm{H}\right), 3227$ 
( $v$ CON-H), $1670(v \mathrm{C}=\mathrm{O}), 1348$ and 1117 ( $\vee \mathrm{S}-\mathrm{N}), 1213$ ( $v \mathrm{C}-\mathrm{O}) \mathrm{cm}^{-1} ; \mathrm{MS}(\mathrm{m} / \mathrm{z}): 349\left(\mathrm{M}^{+}, 15 \%\right), 230(83 \%), 200$ (85\%), 151 (36\%), 135 (42\%), 120 (47\%), 77 (90\%).

Anal. Calcd. for $\mathrm{C}_{15} \mathrm{H}_{15} \mathrm{O}_{5} \mathrm{~N}_{3} \mathrm{~S}: \mathrm{C}, 51.57 \% ; \mathrm{H}, 4.33 \%$; $\mathrm{N}, 12.03 \%$. Found: C, $51.80 \%$; H, $4.77 \%$; N, $11.95 \%$.

\section{6-Methyl-3,4-methylenedioxyphenylsulfonyl-N-4-chlo- rophenylsemicarbazide ( $\underline{10 b})$}

This compound was obtained, in $75 \%$ yield, using 0.11 $\mathrm{mL}$ of 4-chlorophenylisocyanate, as colorless prisms: $\mathrm{mp}$ 201-202 ${ }^{\circ} \mathrm{C} .{ }^{1} \mathrm{H}-\mathrm{NMR}$ (DMSO-d $\left.\mathrm{d}_{6}\right) \delta: 2.50$ (s, $3 \mathrm{H}, \mathrm{ArC}_{3}$ ), $6.9\left(\mathrm{~s}, 2 \mathrm{H}, \mathrm{OCH}_{2} \mathrm{O}\right), 6.95\left(\mathrm{~s}, 1 \mathrm{H}, \mathrm{Ar}-\mathrm{H}_{5}\right), 7.28(\mathrm{~s}, 1 \mathrm{H}$, Ar- $\left.\mathrm{H}_{2}\right), 7.25\left(\mathrm{~d} / \mathrm{J}=8.5 \mathrm{~Hz}, 2 \mathrm{H}\right.$, Ar- $\left.\mathrm{H}_{5}, 3^{\prime}\right), 7.42(\mathrm{~d} / \mathrm{J}=8.5$ $\left.\mathrm{Hz}, 2 \mathrm{H}, \mathrm{Ar}-\mathrm{H}_{2}, 6^{\prime}\right)$ ppm; ${ }^{13} \mathrm{C}-\mathrm{NMR}$ (DMSO-d 6 ) $\delta: 20.08$ $\left(\mathrm{ArCH}_{3}\right), 92.20\left(\mathrm{O}_{\mathrm{CH}} \mathrm{O}\right), 99.71\left(\mathrm{C}_{2}\right), 111.92\left(\mathrm{C}_{5}\right), 120.9$ $\left(\mathrm{C}_{2}\right.$, and $\left.\mathrm{C}_{6^{\prime}}\right), 125.60\left(\mathrm{C}_{4}{ }^{\prime}\right), 128.40\left(\mathrm{C}_{3}\right.$, and $\left.\mathrm{C}_{5^{\prime}}\right), 128.70$ $\left(\mathrm{C}_{6}\right), 134.32\left(\mathrm{C}_{1}\right), 138.31\left(\mathrm{C}_{1}\right), 145.13\left(\mathrm{C}_{3}\right), 150.91\left(\mathrm{C}_{4}\right)$, $154.71(\mathrm{HNC}=\mathrm{O}) \mathrm{ppm} ; \mathbf{I R}(\mathrm{KBr}): 3315\left(v \mathrm{SO}_{2} \mathrm{~N}-\mathrm{H}\right), 3219$ ( $v$ CON-H), $1685(v \mathrm{C}=\mathrm{O}), 1335$ and $119(v \mathrm{~S}-\mathrm{N}), 1221(v$ C-O) $\mathrm{cm}^{-1}$; MS (m/z): $383\left(\mathrm{M}^{+}, 12 \%\right), 230(4 \%), 200$ (90\%), 183 (25\%), 151 (45\%), 135 (55\%), 77 (62\%).

Anal. Calcd. for $\mathrm{C}_{15} \mathrm{H}_{14} \mathrm{O}_{5} \mathrm{~N}_{3} \mathrm{SCl}$ : C, $46.94 \% ; \mathrm{H}, 3.68 \%$; N, 9.95\%. Found: C, 47.03\%; H, 3.80\%; N, $11.05 \%$.

\section{6-Methyl-3,4-methylenedioxyphenylsulfonyl-N-4-bromo- phenylsemicarbazide $(\underline{10 c})$}

This compound was obtained, in $92 \%$ yield, using 0.17 $\mathrm{g}$ of 4-bromophenylisocyanate, as colorless prisms: $\mathrm{mp}$ 214-216 ${ }^{\circ} \mathrm{C} .{ }^{1} \mathrm{H}-\mathrm{NMR}$ (DMSO-d 6 ) $\delta: 2.47$ (s, 3H, $\mathrm{ArC}_{3}$ ), 6.09 (s, $\left.2 \mathrm{H}, \mathrm{OCH}_{2} \mathrm{O}\right), 6.95\left(\mathrm{~s}, 1 \mathrm{H}, \mathrm{Ar}-\mathrm{H}_{5}\right), 7.27(\mathrm{~s}, 1 \mathrm{H}$,

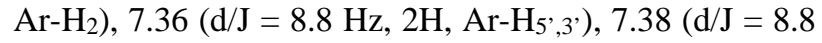
$\left.\mathrm{Hz}, 2 \mathrm{H}, \mathrm{Ar}-\mathrm{H}_{2}, 6^{\prime}\right)$ ppm; ${ }^{13} \mathrm{C}-\mathrm{NMR}$ (DMSO-d ${ }_{6}$ ) $\delta: 20.08$ $\left(\mathrm{ArCH}_{3}\right), 92.17\left(\mathrm{OCH}_{2} \mathrm{O}\right), 99.70\left(\mathrm{C}_{2}\right), 111.91\left(\mathrm{C}_{5}\right), 113.57$ $\left(\mathrm{C}_{4}{ }^{\prime}\right), 120.53\left(\mathrm{C}_{2}\right.$, and $\left.\mathrm{C}_{6}{ }^{\prime}\right), 128.78\left(\mathrm{C}_{6}\right), 131.28\left(\mathrm{C}_{3}\right.$, and $\left.\mathrm{C}_{5}{ }^{\prime}\right), 134.30\left(\mathrm{C}_{1}\right), 138.65\left(\mathrm{C}_{1}{ }^{\prime}\right), 145.09\left(\mathrm{C}_{3}\right) 150.88\left(\mathrm{C}_{4}\right)$, 154.63 (HNCO) ppm; IR (KBr): $3332\left(v \mathrm{SO}_{2} \mathrm{~N}-\mathrm{H}\right), 3225(\mathrm{v}$ CON-H), 1678 ( $v$ C=O), 1328 and $1113(v \mathrm{~S}-\mathrm{N}), 1246(v$ $\mathrm{C}-\mathrm{O}) \mathrm{cm}^{-1}$; MS (m/z): 429 e $428\left(\mathrm{M}^{+}, 18 \%\right), 230$ (6\%), 200 (90\%), 151 (34\%),135 (42\%), 77 (34\%).

Anal. Calcd. for $\mathrm{C}_{15} \mathrm{H}_{14} \mathrm{O}_{5} \mathrm{~N}_{3} \mathrm{SBr}: \mathrm{C}, 42.07 \% ; \mathrm{H}$, $4.14 \%$; N, $11.50 \%$. Found: C, 41.95\%; H, 4.17\%; N, $11.19 \%$.

\section{6-Methyl-3,4-methylenedioxyphenylsulfonyl- $\mathrm{N}$ - phenylthiosemicarbazide ( $\underline{10 d})$}

This compound was obtained, in $80 \%$ yield, using 0.1 $\mathrm{mL}$ of phenylisothiocyanate, as colorless prisms: $\mathrm{mp} \mathrm{163-}$ $165^{\circ} \mathrm{C} .{ }^{1} \mathrm{H}-\mathrm{NMR}$ (DMSO-d $\left.{ }_{6}\right) \delta: 2.50$ (s, 3H, $\left.\mathrm{ArCH}_{3}\right), 6.9$ (s, $\left.2 \mathrm{H}, \mathrm{OC}_{2} \mathrm{O}\right), 6.95\left(\mathrm{~s}, 1 \mathrm{H}, \mathrm{Ar}-\mathrm{H}_{5}\right), 7.30$ (s, $\left.1 \mathrm{H}, \mathrm{Ar}-\mathrm{H}_{2}\right)$, 7.25 (m, 5H, Ar- $\left.\mathrm{H}_{2-6}{ }^{6}\right) \mathrm{ppm} ;{ }^{13} \mathrm{C}-\mathrm{NMR}$ (DMSO-d $\left.{ }^{6}\right) \delta$ : $20.29\left(\mathrm{ArCH}_{3}\right), 92.29\left(\mathrm{OCH}_{2} \mathrm{O}\right), 99.83\left(\mathrm{C}_{2}\right), 111.99\left(\mathrm{C}_{5}\right)$, $122.11\left(\mathrm{C}_{4}{ }^{\prime}\right), 125.07\left(\mathrm{C}_{2}\right.$, and $\left.\mathrm{C}_{6}\right), 128.00\left(\mathrm{C}_{3}{ }^{\prime}\right.$ and $\left.\mathrm{C}_{5}{ }^{\prime}\right)$,
$128.22\left(\mathrm{C}_{6}\right), 134.79\left(\mathrm{C}_{1}\right), 138.78\left(\mathrm{C}_{1}\right), 145.32\left(\mathrm{C}_{3}\right), 151.21$ $\left(\mathrm{C}_{4}\right), 180.86(\mathrm{HNC}=\mathrm{S}) \mathrm{ppm}$; IR $(\mathrm{KBr}): 3289\left(v^{\mathrm{SO}} \mathrm{O}_{2} \mathrm{~N}-\mathrm{H}\right)$, 3215( $\vee$ CSN-H), 1665 ( $\vee \mathrm{C}=\mathrm{S}), 1329$ and $1112(v \mathrm{~S}-\mathrm{N})$, 1227 ( $\mathrm{v} \mathrm{C}-\mathrm{O}) \mathrm{cm}^{-1}$; MS (m/z): $349\left(\mathrm{M}^{+}, 12 \%\right), 230(7 \%)$, 200 (85\%), 135 (45\%), 120 (47\%), 77 (90\%).

Anal. Calcd. for $\mathrm{C}_{15} \mathrm{H}_{15} \mathrm{O}_{4} \mathrm{~N}_{3} \mathrm{~S}_{2}$ : C, $49.30 \% ; \mathrm{H}, 4.14 \%$; N, $11.50 \%$. Found: C, 48.9\%; H, 4.45\%; N, 9.78\%.

\section{Biological}

\section{Platelet Aggregation}

Blood was collected from rabbits by puncture of the central ear artery into $3.8 \%$ sodium citrate $(9: 1 \mathrm{v} / \mathrm{v})$. Platelet-rich plasma (PRP) was prepared by centrifugation, 500 $\mathrm{x} \mathrm{g}$ for $9 \mathrm{~min}$, at room temperature, and platelet count was adjusted to $5 \times 10^{8}$ platelets $/ \mathrm{mL}$.

Platelet aggregation was monitored by the turbidimetric method ${ }^{25}$ in a Chrono-Log aggregometer. PRP $(400 \mu l)$ was incubated at $37{ }^{\circ} \mathrm{C}$ for $1 \mathrm{~min}$. with continuous stirring at $900 \mathrm{rpm}$ and then stimulated with ADP $(5 \mu \mathrm{M}$ in distilled water), collagen ( $5 \mu \mathrm{g} / \mathrm{mL}$ in saline), arachidonic acid (AA - $200 \mu \mathrm{M}$ in ethanol) or U-46619 (3 $\mu \mathrm{M}$ in ethanol).

Test compounds (10a-d) and the vehicle ( $0.5 \%$ DMSO, $2 \mu \mathrm{L}$ ) were added to the PRP samples $5 \mathrm{~min}$. before addition of the aggregating agent. The DMSO used as vehicle did not have either pro- or antiplatelet aggregation activity. Indomethacin $(9 \mu \mathrm{M})$, a classical cyclooxygenase inhibitor, was used as standard.

The platelet aggregation was expressed as percentage of aggregation for ADP, AA, U-46619 and as the maximum rate of aggregation (slope) for collagen. Data were analyzed statistically by Student's " $\mathrm{t}$ " test for a $p$ value of $<0.05$ and are expressed as mean $\pm \mathrm{SD}$. for $\mathrm{n}$ experiments in triplicate.

\section{Acknowledgments}

We are grateful to CNPq (BR., grant \#52.0033/96-5), Pronex (BR, FINEP 0888) and FUJB (BR., grant \#6276-6; \# 6487-4 and \#7675-9) for the financial support of this work and to CNPq, FAPERJ and CAPES (BR.) for fellowships (LML, CBO, ALPM, CAMF and EJB).

\section{References}

1. Higgs, G.A.; Higgs, E.A.; Moncada, S. Compreenhesive Medicinal Chemistry 1990, 2, 1147-1157.

2. Gresele, P.; Arnout, J.; Deckmyn, H.; Huybrechts, E.; Pieters, G.; Vermylen, J. J. Clin. Invest. 1987, 80, 1435-1445.

3. Hamberg, M.; Svensson, J.; Samuelsson, B. Proc. Natl. Acad. Sci. U.S.A. 1975, 72, 2994-2998.

4. Ogletree, M.L. Fed. Proc. 1987, 46, 133-138.

5. Hirata, M.; Hayashi, Y.; Ushikubi, F.; Yokota, Y.; Kageyama, R.; Nakanishi, S.; Narumiya, S. Nature 1991, 349, 617-620. 
6. Kin, S-O.; Lim, C.T.; Lam, S.C-T.; Hall, S.E.; Komiotis, D.; Venton, D.L.; Le Breton, G.C. Biochem. Pharmacol. 1992, 43, 313-322.

7. Hall, S.E. Med. Res. Rev. 1991,11, 503-579.

8. Collington, E.; Finch, H. Annu. Rept. Med. Chem. 1990, 25, 99-98.

9. Ackerley, N.; Brewster, A.G.; Brown, G.R.; Claeke, D.S.; Foubister, A.J.; Griffin, S.J.; Hudson, J.A.; Smithers, M.J.; Whittamore, P.R.O. J. Med. Chem. 1995, 38, 1608-1628.

10. Komiotis, D.; Lim, C.T.; Dietis, J.P.; Le Breton, C.G.; Venton, D.V. J. Med. Chem. 1992, 35, 3033-3039.

11. Misra, R.N.; Brown, B.R.; Han, W.C.; Harris, D.N.; Hedberg, A.; Webb, M.L.; Hall, S.E. J. Med. Chem. 1991, 34, 2882-2891.

12. Hagashita, S.; Seno, K. Chem. Pharm. Bull. 1989, 37, 327-335.

13. Stegmeier, K.; Phill, J. Patscheke, H.; Arch. Pharmacol. 1986, 322, 1-3.

14.Sato, M.; Kawashima, Y.; Goto, J.; Yamane, Y.; Chiba, Y.; Jinno, S.; Satake, M.; Iwata, C. Eur. J. Med. Chem. 1995, 30, 403.

15. Sakurai, S.; Ogawa, N.; Suzuki,T.; Kato, K.; Ohashi, T.; Yasuda, S.; Kato, H.; Ito, Y. Chem. Pharm. Bull. 1996, 44, 765.
16. Lima, L.M.; Brito, F.F.; Ormelli, C.B.; Miranda, A.L.P.; Fraga, C.A.M.; Barreiro, E.J. Pharm. Acta Helv. 1999, 73, 281-92.

17. Barreiro, E.J.; Costa, P.R.R.; Coelho, F.A.S.; Farias, F.M.C. J. Chem. Res. (M) 1985, 2301-2332.

18. Lages, A.S.; Silva, K.C.M.; Miranda, A.L.P.; Fraga, C.A.M.; Barreiro, E.J. Bioorg. Med. Chem. Lett. 1998, 8, 183-188.

19. Fraga, C.A.M. MSc. Thesis, Universidade Federal do Rio de Janeiro, BR, 1991.

20. Fraga, C.A.M.; Barreiro, E.J. J. Heterocycl. Chem. 1992, 29, 1667-1669.

21. Silva, E.F.; Barreiro, E.J. J. Braz. Chem. Soc. 1993, 4, 40-44.

22. Barreiro, E.J.; Lima, M.E.F. J. Pharm. Sci. 1992, 81, 1219-1222.

23. Boschelli, D.H.; Connor, D.T.; Bornemeier, D.A.; Dyer, R.D., Kennedy, J.A.; Kuipers, P.J.; Okonkwo, G.O.; Schrier, D.J.; Wright, C.D. J. Med. Chem. 1993, 36, 1802-1809.

24. Lewis, G.P.; Watts, I.S. Br. J. Pharmacol. 1982, 75, 623-631.

25. Born, G.V.R.; Cross M.J. J. Physiol. 1963, 168, 178181. 